\title{
Atención y Cuidado de la Primera Infancia en México: Un Reto para la Equidad de Género
}

\author{
Early Childhood Care in Mexico: A Challenge for Gender \\ Equality
}

\author{
Claudia Alaníz-Hernández * \\ Universidad Pedagógica Nacional, México
}

\begin{abstract}
El presente trabajo busca abonar a la reflexión respecto de las políticas de cuidado de la primera infancia en México. Se basa en el análisis de la Estrategia Nacional de Atención para la Primera Infancia (ENAPI 2020) del gobierno del presidente Andrés Manuel López Obrador en el marco del establecimiento de la obligatoriedad de la Educación Inicial para la niñez mexicana. Metodológicamente se consideran como base las dimensiones de Muller y Surel (1998) para el análisis de las políticas públicas: a) Toma de Decisión, b) Reglas y c) Interacciones. En ellas, donde se expresa la concepción del derecho de la infancia, su cuidado y las implicaciones que tiene en el cruce con la desigualdad de género. Entre los hallazgos se encontró que la Estrategia Nacional para la Atención de la Primera Infancia en México se caracteriza por su heterogeneidad y fragmentación, además que presenta un sesgo familiarista, sin reconocer que el cuidado infantil en los primeros años se ha convertido en un factor de desigualdad, vinculado fuertemente a las relaciones de poder y el género; con ello subsiste una visión conservadora que coloca el cuidado de las niñas y niños en el ámbito de la vida privada, reproduciendo los estereotipos de género que terminan por afectar la equidad de manera contradictoria, al reafirmar la responsabilidad cuasi natural y primordial de las mujeres en el cuidado infantil, limitando con ello su autonomía económica.
\end{abstract}

Descriptores: Infancia; Política; Derechos; Bienestar; Justicia social.

\begin{abstract}
This paper seeks to reflect on the early childhood care policies in Mexico. It is based on analysis of the National Strategy for Early Childhood Care (ENAPI, 2020) by President Andrés Manuel López Obrador's government, within the framework of the establishment of the compulsory Initial Education for Mexican Childhood. Methodologically, Mueller \& Surel's (1998) dimensions for public policies analysis are considered: a) Decision making, b) Rules and c) interactions. Childhood rights conception, its care and the implications it has on gender inequality are expressed on those policies. Among the findings was found National of Early Childhood Attention Strategy in Mexico is characterized by its heterogeneity and fragmentation, in addition it has a familiarity bias, without recognizing that child care on the early years has become an inequality factor, strongly linked to power relations and gender with this a conservative vision subsists which places children's care to the scope of the private life reproducing gender stereotypes that end up affecting equity in a contradictory way by reaffirming responsibility quasi natural and primordial of women in child care, thereby limiting their economic autonomy.
\end{abstract}

Keywords: Childhood; Policy; Rights; Wellness; Social justice.

*Contacto: calaniz@upn.mx

ISSN: 2254-3139

www.rinace.net/riejs/

revistas.uam.es/riejs
Recibido: $\quad 20$ de abril 2020

$1^{\text {a }}$ Evaluación: 27 de julio 2020

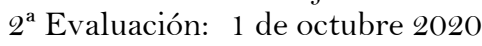

Aceptado: 15 de octubre 2020 


\section{Introducción}

Por las características propias de la primera infancia y la trascendencia de su adecuada atención y cuidado, constituyen un asunto de interés general a incorporar en la política pública, entendida como un conjunto de acciones decididas por el gobierno (en interlocución e interacción con la sociedad), a fin de atender un problema o realizar un objetivo de interés público (Aguilar, 2015). La atención de las niñas y niños pequeños recién se abre camino dentro de las políticas de cuidado, por lo que se aborda no sólo con el propósito de alcanzar niveles de bienestar social, sino como condición clave para atender la desigualdad de género.

Como objeto de estudio, el cuidado tiene su antecedente en las críticas al capitalismo desde la ideología marxista (por la división sexual del trabajo) y la subordinación del trabajo reproductor o doméstico realizado por las mujeres, al trabajo remunerado de los hombres. Por otro lado, se puede ubicar el surgimiento del tema del cuidado infantil en los debates feministas de los años setenta, a partir del trabajo de Meillasoux (1975), quien identificó, además de la división sexual del trabajo, su doble función: productiva para los hombres (remunerada y asociada con la acumulación de capital), y reproductiva de la moral social para las mujeres (relacionada al cuidado de la familia y con el trabajo no remunerado). "Desde esta perspectiva, el proceso de mercantilización del trabajo escindió los ámbitos de lo productivo y lo reproductivo" (Florit y Piedracueva, 2016, p. 2). Así, la lucha feminista evidenció el carácter patriarcal del capitalismo, donde el cuidado quedó anclado a la división sexual del trabajo reproductivo de la mujer y colocado dentro del ámbito privado de lo familiar y lo doméstico.

Su abordaje como políticas de tiempo surge a finales de 1990 a partir de la propuesta de ley de iniciativa popular le donne cambiano il tempo, de las mujeres del entonces Partido Comunista en Italia, abordándola desde "desde la centralidad de los cuidados y la satisfacción de las necesidades humanas a partir de una mirada situada (y) sensible a las dinámicas del día a día” (Legarreta, 2014, p. 94). Si bien no llegó a aprobarse, marcó un hito en la definición hegemónica del tiempo como problema político, que da estructura a la vida social, y en el papel de las políticas púbicas como factor clave para el cambio social. Dicha postura alimentó debates y proyectos en toda Europa, pues evidenció el vínculo de la economía con el género y su sesgo patriarcal.

La crítica logró su cometido porque en 1995 la Declaración y Plataforma de Acción de Beijing, se definió a favor de los derechos de las mujeres y las niñas en todo el mundo. Con ello empezó a medirse el tiempo dedicado en el trabajo reproductivo o doméstico. Pero este avance se enfocó más en el trabajo doméstico que en las actividades relacionadas con el cuidado infantil, conservándolo dentro del estereotipo de acción propia de la naturaleza femenina.

Paulatinamente el cuidado se incorporó dentro de las mediciones de tiempo, aunque algunas voces han cuestionado la pretensión de medirlo linealmente en horas, pues deja fuera las dimensiones relacionales y motivacionales del cuidado (Esquivel, 2011a; Folbre,1995; Himmelweit, 1995; Legarreta, 2014). Nos referimos al cuidado como "las actividades y relaciones orientadas a alcanzar los requerimientos físicos y emocionales de niños y adultos dependientes, así como los marcos normativos, económicos y sociales dentro de los cuales éstas son asignadas y llevadas a cabo" (Daly y Lewis, 2000, p. 285). 
De cualquier forma, constituye un avance al visibilizar el tiempo de trabajo no remunerado que implica el cuidado.

Se entiendo por primera infancia el "periodo de la vida, de crecimiento y desarrollo comprendido desde la gestación hasta los 7 años aproximadamente" (Jaramillo, 2007, p. 110), pero dentro de las políticas el corte de edad se asocia al ingreso a la educación básica o primaria y en el caso de Educación Inicial se considera hasta los 3 o 4 años de edad. Como objeto de estudio, las Políticas de Atención y Educación de la Primera Infancia (PAEPI) en América Latina son un campo de investigación "delimitado por la tríada desarrollo - inversión - derecho" según la clasificación de Presno, López y Moya (2019, p. 10); es decir, la mayor parte de las investigaciones están orientadas al desarrollo infantil y su importancia para alcanzar el potencial y aprendizaje a lo largo de toda la vida; un segundo eje conceptual se apoya en las teorías del capital humano (Gómez, García y García, 2019), donde se reconoce que existe un menor costo en la inversión en la primera infancia en comparación con los recursos requeridos para la atención posterior del rezago y reinserción; y el tercero, corresponde a estudios sobre el marco jurídico de los derechos de niñas y niños. Argentina muestra una larga trayectoria de estudios sostenidos respecto al cuidado infantil, en contraste, México es uno de los países latinoamericanos (junto con Bolivia, Costa Rica, Ecuador y Perú) con una producción incipiente de estudios. En ellos pareciera apenas iniciar "estas discusiones, y se encuentra en proceso de dar nuevos debates en relación al cuidado como derecho, la calidad de las prestaciones (y) la evaluación del carácter intersectorial de las políticas (en pos de la integralidad)" (Presno, López y Moya, 2019, p. 8). Varios de los trabajos existentes se cruzan con la agenda de género y el trabajo de las mujeres, por una asociación cultural patriarcal de supuestas cualidades femeninas para realizar acciones de cuidado.

Dichos estudios son elaborados por académicos, instancias gubernamentales y organismos internacionales y han logrado paulatinamente posicionar el cuidado y atención de la primera infancia en la agenda global. Tan es así, que la Organización Mundial de la Salud establece: "Los gobiernos deben reconocer que las inversiones adecuadas en la primera infancia representan la piedra angular del desarrollo humano y son esenciales para el progreso de las sociedades" (Irwin, Arjumand y Hertzman, 2007, p. 21).

En América Latina encontramos, fundamentalmente, tres tipos de políticas de cuidado: a. Las asistenciales (de corto plazo); b. Sistémicas e integrales (vinculan los sectores de educación y trabajo); y c. Políticas para un nuevo pacto social: orientadas a cuestionar la división sexual del trabajo y a promover el desarrollo sostenible, donde el eje del cuidado es central para el desarrollo. La mayoría de los países se ubica en el primer caso; como ejemplo de las segundas, la República Bolivariana de Venezuela, el Ecuador, el Estado Plurinacional de Bolivia y la República Dominicana han incluido el reconocimiento del trabajo no remunerado de las mujeres en sus constituciones políticas. En Uruguay, Costa Rica, Ecuador, Jamaica y Surinam, las necesidades de cuidado se están perfilando como un campo específico de políticas públicas. Para el tercer tipo, solo hay tres casos emblemáticos en la región: "Chile Crece Contigo," "De Cero a siempre" (Colombia) y "Educa a tu Hijo" (Cuba), donde prevalece la convicción de garantizar el derecho de la infancia.

Los debates sobre el cuidado pretenden visibilizar el tiempo dedicado al trabajo que implica la atención de dependientes, particularmente niñas y niños (aunque también pueden ser adultos mayores, enfermos y personas con discapacidad), para sacarlo del ámbito privado de la vida familiar (y de la asociación a la naturaleza femenina), 
posicionarlo en el terreno de la política social (por sus implicaciones económicas) y transferir la responsabilidad del cuidado de la familia al Estado. Pero México (al igual que Argentina, Brasil y Costa Rica) por lo menos hasta el año 2018 mantuvo un sesgo familiarista en el cuidado infantil, y aunque cada vez se observa mayor participación de las mujeres en el trabajo remunerado, no hay mecanismos para que los hombres participen más en las actividades no remuneradas dentro del hogar.

Por ello la necesidad de que el Estado asuma la responsabilidad de proveer servicios de cuidado infantil, para romper la reproducción de los estereotipos de género en la división sexual del trabajo. Por ejemplo, el arribo de gobiernos progresistas en América Latina posicionó en la agenda pública la protección social, aunque de manera diferenciada, privilegiando la política social como tema relevante. Varios países fueron incorporado el cuidado infantil a la política pública con mayor participación de los hombres, extendiendo las licencias de paternidad que varían de 2 a 45 días en la región: Argentina y Paraguay dos días, el Estado Plurinacional de Bolivia tres días, la República Bolivariana de Venezuela 14 días, Costa Rica y Colombia 15 días, Perú y Puerto Rico conceden 4 a 8 días, Ecuador 10 días y en Chile mes y medio.

El presente artículo retoma las tres dimensiones de Muller y Surel (1998) para el análisis de las políticas públicas: a) Toma de Decisión, b) Reglas y c) Interacciones, con el propósito de revisar la Estrategia Nacional de Atención Infantil en México (ENAPI) lanzada en 2020 que comprende la atención de niñas y niños de 0 a 3 años de edad y su incorporación al esquema de educación básica, en el marco de la reforma constitucional al Artículo tercero que estableció la obligatoriedad de la educación inicial en mayo de 2019, lo que implicaría pasar de la concepción tutelar de la infancia a la garantista de sus derechos por parte del Estado mexicano.

El cuidado infantil de los primeros años va más allá de las necesidades vitales (hambre, sueño, alimentación y aseo), salud y educación; implica también la satisfacción de las necesidades emocionales y relacionales, además de ser el periodo de vida ideal para potenciar su desarrollo integral a lo largo de toda la vida; es decir, proveer el bienestar de las niñas y niños. De ahí la importancia de fortalecer su atención integral; no en balde existen profesiones ligadas a la estimulación y desarrollo de la infancia, aunque persista una descalificación social estructural del trabajo de cuidado infantil al estar feminizado por considerarlo no experto y asistencial. Esta visión ha sido el principal obstáculo de la Educación Inicial. La respuesta a la pregunta de en quién debe recaer la responsabilidad de atender las necesidades de cuidado de bebés, infantes y niños pequeños depende, en principio, de construcciones ideológicas y sociales: en las ideologías conservadoras sería claro situarlo dentro del ámbito privado del hogar y atribuirlo a las actividades propias de la mujer como respuesta natural; pero dentro de sociedades democráticas o progresistas se ubica dentro del ámbito público, por lo que son el Estado y la sociedad quienes deben adjudicarse esa corresponsabilidad al asumirlo dentro de la agenda de políticas públicas.

Existen múltiples problemas asociados con la atención y el cuidado de la primera infancia: la imposibilidad de que los propios sujetos exijan sus derechos, la fragmentación de las acciones, la falta de personal especializado para la construcción de estrategias intersectoriales, la escasa profesionalización del personal que atiende directamente a las y los bebés (0-1 año), infantes (1-2 años) y las niñas y niños pequeños (2-3 años), aunado a los problemas estructurales de desigualdad, discriminación y pobreza, que los coloca como uno de los grupos sociales más vulnerables, pues ya se ha documentado que la pobreza 
tiene rostro de mujer, particularmente de niña (CEPAL, 2004). Por si ello fuera poco, también existe una visión adultocentrista y patriarcal que mira la infancia como el futuro y no como grupo social con características y necesidades propias: incluso está a debate el mismo concepto de infancia por la connotación de minusvalía en términos históricos y culturales, ante la diversidad de formas en que se vive la niñez en la actualidad. Esta ausencia se observa en los documentos que no expresan abiertamente un concepto de niñez referida al "período vital reservado al desarrollo psicobiológico y social" (Alzate, citado en Jaramillo, 2007, p. 112). Para lograrlo se requiere el respaldo de un marco institucional y políticas públicas articuladas para garantizar el derecho a la autonomía económica de las mujeres y los derechos humanos de la niñez bajo el principio de ciudadanía.

\section{Atención a la primera infancia: Entre el ámbito familiar/privado y el social/público}

De acuerdo con Muller y Surel (1998), la primera dimensión a analizar en la definición de las políticas públicas corresponde a la toma de decisiones. Para el caso de la atención de la niñez en sus primeros años de vida, es necesario retomar como antecedente que, dentro de la división sexual del trabajo, el cuidado fue parte del trabajo natural de la mujer que "parecía diluirse mágicamente en el aire, por cuanto no arrojaba un producto económicamente visible como el del hombre" (Larguía y Dumoulin, 1976, p. 18). Con ello se invisibilizaron en la atención familiar y las tareas dirigidas a los bebés, infantes y niños pequeños tales como la socialización temprana, su cuidado y la transmisión de normas sociales. Así, la visión patriarcal permeó en la institucionalización de las políticas públicas destinadas a la infancia, bajo dos concepciones:

- La tutelar, basada en las nociones de la infancia como propiedad privada, pasiva, inmadura e incompleta, cuyo valor consiste en alcanzar un futuro.

- La garantista, de protección integral derivada de la Convención de los Derechos del Niño. Como principio epistemológico y ético, concibe a niñas y niños como sujetos plenos de derecho y es capaz de generar nuevas prácticas.

La base de la doctrina tutelar... al igual que el androcentrismo en la cultura patriarcal requiere de diversas medidas y acciones para lograr cambios. La importancia de dicha orientación radica en que de ella depende la racionalización, se construyen métodos y técnicas de intervención social, política y jurídica que centran sus objetivos en el protagonismo infantil y el cumplimiento de estos derechos en todos los ámbitos (Colín, 2014, p. 60).

La reducción del cuidado infantil a las tareas domésticas condenó a las mujeres a realizar un trabajo no remunerado que las instaló en una condición de dependencia económica y, por tanto, proclive a la pobreza al no disponer de recursos propios. Es por ello que los feministas más radicales impulsaron que las encuestas económicas fueran ponderando en sus mediciones el cuidado y las actividades domésticas como trabajo no remunerado.

Tres situaciones sociales se deben considerar como factores que afectan las actividades del cuidado: por un lado, la incorporación de las mujeres al mercado de trabajo remunerado; por otro, la modificación del modelo tradicional de familia; y el tercero se relaciona con la reproducción estructural de la pobreza. "Es sabido que el tamaño medio de los hogares es mayor en los sectores de ingresos más bajos, dada la mayor fecundidad y la presencia 
mayor de tres generaciones (una abuela conviviente)" (Esquivel, Faur y Jelin, 2014, p. 23). Esto es consistente en México, donde existen más familias extendidas en condición de pobreza (figura 1).

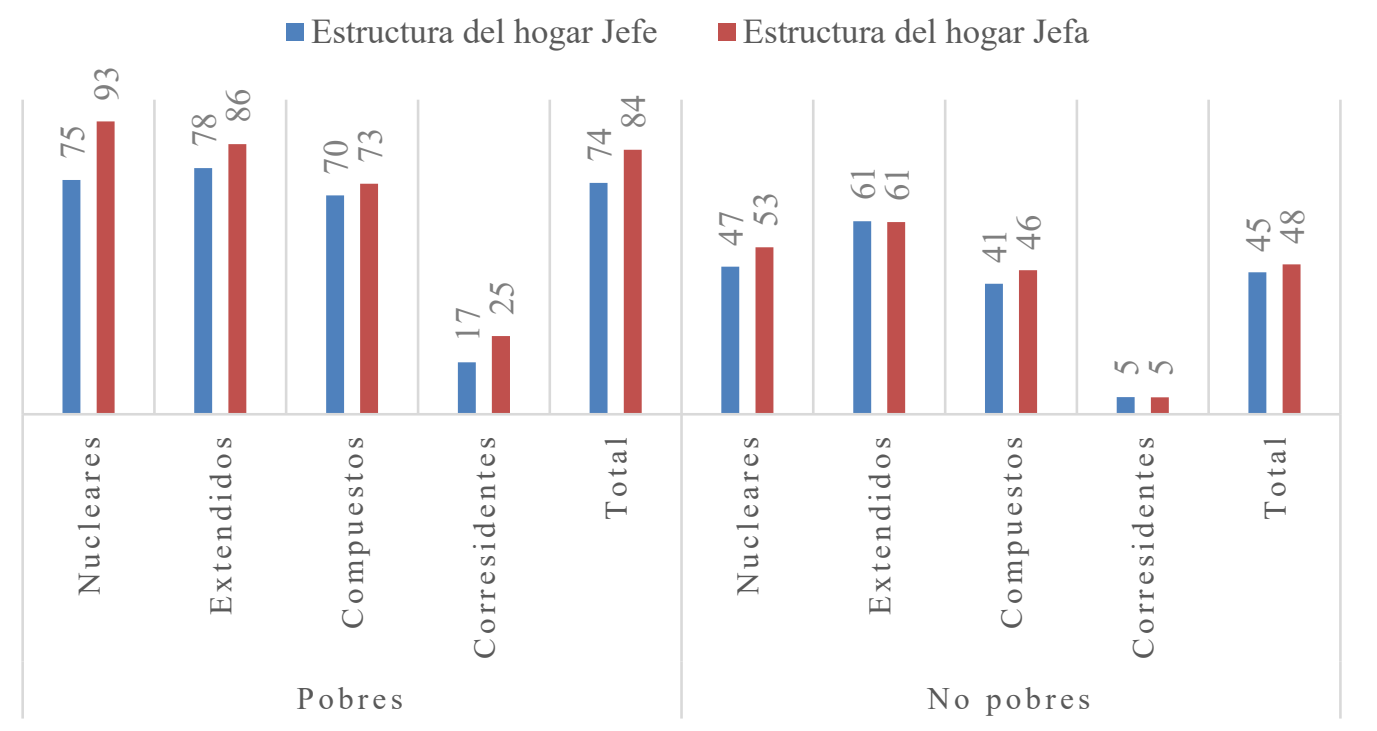

Figura 1. Dependencia demográfica promedio del hogar, según estructura, sexo y condición de pobreza de la jefatura (2018)

Fuente: Base de Datos y Publicaciones Estadísticas de la CEPAL (2020).

$\mathrm{El}$ ingreso de las mujeres al mercado laboral puso en crisis el sistema social de cuidado infantil, lo visibilizó y se vinculó con los derechos laborales de la mujer como parte de sus prestaciones, pero se mantuvo básicamente desde una concepción tutelar. Dicha perspectiva permitió exentar el cuidado de las responsabilidades del Estado (salvo la históricamente adjudicada vinculada a la orfandad y a la caridad). Por un lado, trajo consigo políticas y programas de "conciliación" (Faur, 2014), destinados a que las mujeres hagan compatible su responsabilidad del cuidado infantil, el trabajo del hogar y el remunerado, con participación marginal de los hombres (por ejemplo, días de cuidado al nacimiento de los hijos o servicio de guardería cuando la madre está ausente), sin alcanzar a transitar a una concepción garantista del derecho de la niñez.

Por otro lado, esta acotación de la participación gubernamental generó un nicho de mercado que se ha expandido en la medida que las mujeres continuaron incorporándose al trabajo remunerado. El cuidado infantil se constituyó al mismo tiempo en actividad remunerada y sujeta a la regulación del Estado. Pero el problema radica en que no termina por asumirse como un asunto de interés público para ser atendido desde la política pública, desde una concepción garantista del derecho del niño al desarrollo pleno y como un asunto de interés social/público. Por eso se mantiene dentro del ámbito familiar/privado.

Dicha concepción se modifica cuando se incorpora la noción de bienestar como propósito último de la política social, la "articula además (con) la provisión de otras instituciones, como el mercado de trabajo y las familias que inciden en las oportunidades y la calidad de vida de la población" (Esquivel, Faur y Jelin, 2014, p. 29). Con ello es evidente la decisión del Estado al posicionarse o deslindarse de la responsabilidad del cuidado, impacta en la calidad de vida de las mujeres, las niñas y los niños al posibilitar el ejercicio pleno de sus 
derechos. El cuidado requiere un entramado institucional que articule trabajo, seguridad social y educación inicial; de lo contrario la deja en manos del mercado.

Es justamente desde el punto de vista de la producción de bienestar que una amplia literatura elaborada en los países desarrollados ha utilizado el concepto de "cuidado" como categoría de análisis de los "estados de bienestar". Debido a que el cuidado se encuentra en la intersección entre las relaciones sociales y de género en los que los Estados, a través de sus políticas, sobreimprimen en ellas las responsabilidades de proveerlo, el cuidado se transforma, desde esta perspectiva, en una dimensión desde la cual analizar las políticas sociales (Daily y Lewis, 2000 citado en Esquivel, 2011b, p. 16).

La atención de la primera infancia ha permanecido casi invisible dentro del ámbito familiar, sin ponderar que es determinante en la vida de todo individuo, pues en los primeros cinco años se establecen las bases para el desarrollo físico, motor, socioemocional, cognitivo y de lenguaje. Para garantizar un desarrollo pleno, es necesario mejorar las condiciones de salud, nutrición, protección, cuidado y estimulación, para que se dé el aprendizaje a lo largo de toda la vida. De lo contrario, las carencias en la primera infancia generan consecuencias irreversibles.

Este derecho reconocido e incluido en pactos y tratados internacionales, aún está en "construcción" desde el punto de vista de su exigibilidad e involucra diferentes aspectos de gran importancia. En primer lugar, el derecho a recibir los cuidados necesarios en distintas circunstancias y momentos del ciclo vital, evitando que la satisfacción de esa necesidad se determine por la lógica del mercado, la disponibilidad de ingresos, la presencia de redes vinculares o lazos afectivos. En segundo lugar, y esta es quizás la faceta menos estudiada, el derecho de elegir si se desea o no cuidar en el marco del cuidado familiar no remunerado; se trata de no tomar este aspecto como una obligación sin posibilidad de elección durante toda la jornada. Refiere, por tanto, a la posibilidad de elegir otras alternativas de cuidado que no sean necesariamente y de manera exclusiva el cuidado familiar no remunerado.

Como plantea Pautassi (2010, 2012), para algunos actores sociales y políticos el cuidado es simplemente una prestación dirigida a las mujeres que buscan trabajar, bajo la falacia de que se debe "apoyar a las mujeres" que necesiten o quieran trabajar. Por el contrario, desde la perspectiva de derechos, el cuidado es un derecho de todos y debe garantizarse mediante los arreglos institucionales y presupuestarios que se requieran y debe ser normado y apoyado por el Estado. No es un beneficio para las mujeres y sí un derecho de quienes lo requieren. Así, el cuidado infantil como uno de los campos de la protección social que debe ser resuelto desde la ecuación institucional Estado-empresas-familiastercer sector (Montaño y Calderón, 2010).

\subsection{Los primeros años de vida en México}

La segunda dimensión de análisis corresponde al marco normativo. En México desde la constitución de 1917 el derecho al cuidado infantil se encuentra supeditado al derecho laboral de las mujeres, y éste a la condición de empleo formal. Quienes tienen acceso a seguridad social cuentan con una licencia con goce de sueldo de seis semanas anteriores y seis posteriores a la fecha de parto; dos descansos de 30 minutos al día para atender la lactancia de sus hijos por un periodo de seis meses y derecho a centros de atención infantil de cero a cuatro años de su hijo. 
El primer problema al respecto es que comparativamente es bajo el porcentaje de mujeres que tienen empleo remunerado, y de ellas un sector importante labora en la informalidad sin acceso a la seguridad social e incluso hay quienes no reciben remuneración por su trabajo. La falta de empleo formal y escasa protección social ha permitido que el número de guarderías y centros de atención infantil no crezca. En 2017 todas las modalidades de empleo informal sumaron 29.1 millones de personas, lo que representa que casi el $60 \%$ de la población económicamente activa carece de seguridad social (Cruz, 2017). Esta situación ayuda a explicar el escaso crecimiento de la educación inicial en el país.

Hasta el año 2000 se aprobó la Ley para la Protección de los Derechos de Niñas, Niños y Adolescentes. Sin embargo, no se diseñaron los mecanismos para garantizar su aplicación y los asuntos relacionados con la atención de la infancia continuaron a cargo del Sistema Integral de Atención a la Familia (DIF). Fue hasta 2014 cuando se publicó la Ley General de Protección a los Derechos de las Niñas, los Niños y los Adolescentes (LGDNNA) que se creó el Sistema de Protección Integral (SPINNA), con el propósito de articular las acciones en los diferentes niveles de gobierno (federal, estatal y municipal), en todo el territorio nacional y con la confluencia de todos los actores involucrados (públicos, privados y sociedad civil).

Pero el siguiente panorama refleja el escaso respeto al derecho de las niñas y los niños. En México viven 12,7 millones de infantes menores de 5 años. El 18\% no alcanza su desarrollo óptimo, según el informe 2017 del Fondo de la Naciones Unidas para la Infancia (UNICEF por sus siglas en inglés), 2 de cada 10 niñas y niños menores de 5 años en zonas rurales presentan desnutrición crónica y afecta al $25 \%$ de los niños que viven en hogares indígenas. En contraste, 9,7\% de los niños menores de 5 años padecen obesidad (México ocupa el primer lugar en obesidad infantil en el mundo). Los otros factores asociados al inadecuado desarrollo infantil son la pobreza (la mitad de los niños de 0 a 5 años de edad viven en condición de pobreza y el $20 \%$ en pobreza extrema); la falta de libros o la no asistencia a programas educativos y de interacción en actividades que promueven su aprendizaje (leer, cantar, jugar o dibujar con y para ellos). Además, faltan mecanismos de protección contra el maltrato, $5.1 \%$ de los niños viven en condiciones inadecuadas para su desarrollo (de nutrición, protección y estimulación) y 1 de cada 2 niños sufre alguna forma de violencia dentro de su hogar.

A pesar de ese panorama, la falta de obligatoriedad permitió que durante el ciclo escolar 2017-2018 solamente asistieran 95.766 infantes a algún Centro de Educación Inicial (0 a 3 años) a nivel nacional: 62,2\% en algún subsistema de sostenimiento público y el resto en el sector privado (Secretaría de Educación Pública, 2020).

Lo anterior es consecuencia de que $60 \%$ de la infancia mexicana no cuenta con seguridad social (figura 2), es decir: viven del empleo informal de sus padres sin protección social alguna; $56,6 \%$ de ellos se ubica en la zona centro del país (Ciudad de México, Estado de México, Guerrero, Morelos, Puebla, San Luis Potosí y Tlaxcala); y 68,9\% en el sureste (que comprende los estados de Campeche, Chiapas, Oaxaca, Quintana Roo, Tabasco, Veracruz y Yucatán) (Consejo Nacional de Evaluación de la Política de Desarrollo Social, 2018a). 


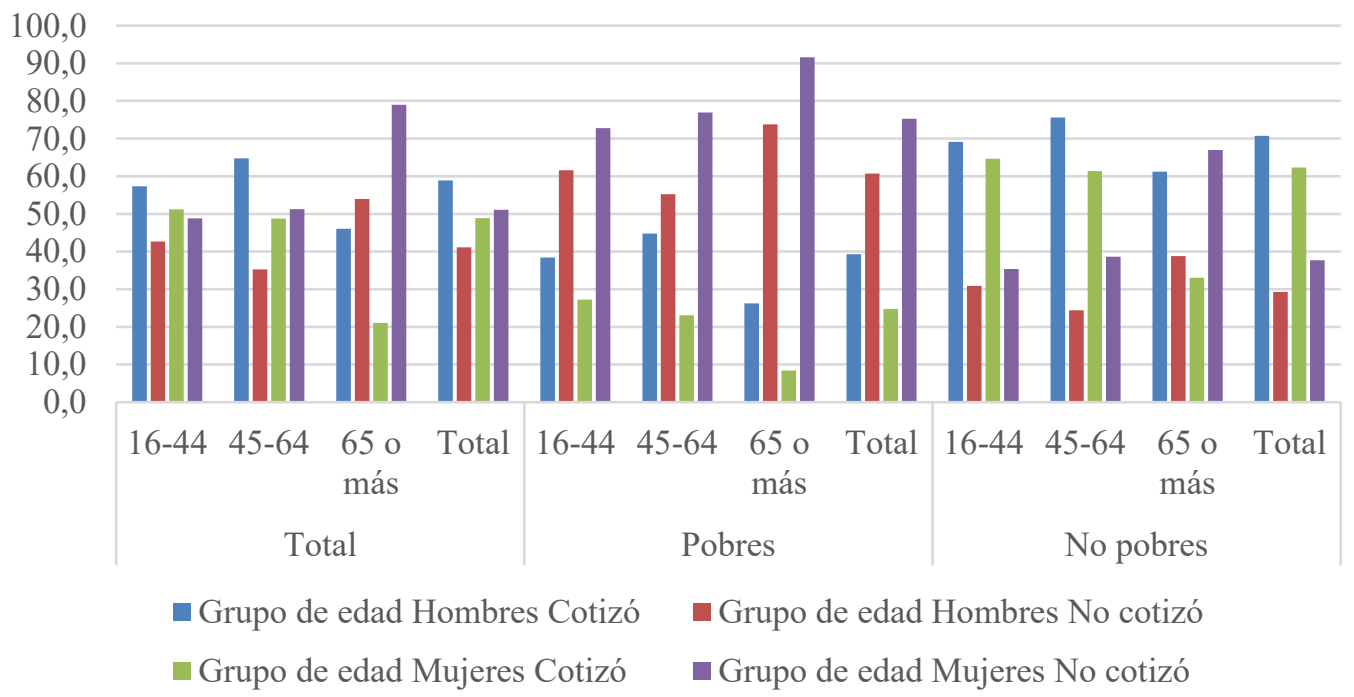

Figura 2. Porcentaje de hombres y mujeres ocupados según cotización a la seguridad social, por grupo de edad y condición de pobreza 2008-2018

Fuente: Base de Datos y Publicaciones Estadísticas de la CEPAL (2020).

En México, la geografía rural e indígena, así como las características del hogar, determinan desde el momento de su nacimiento, el nivel de pobreza en que vivirán las niñas y niños. Por ello, las comunidades rurales indígenas de tres estados: Oaxaca, Guerrero y Chiapas, concentran 95\% de la infancia en situación de pobreza (Consejo Nacional de Evaluación de la Política de Desarrollo Social, 2018b).

Los componentes que determinan la condición de pobreza de la infancia en México a partir de los estudios del Consejo Nacional de Evaluación de la Política de Desarrollo Social (CONEVAL) son: el número de habitantes en el hogar (mayor propensión en hogares de más de 5 integrantes); acceso a servicios mínimos como agua, drenaje y electricidad; el número de adultos con trabajo remunerado; escolaridad de la madre (a menor escolaridad mayor nivel de pobreza) y tipo de empleo, pues el informal determina la privación el acceso a servicios de salud, programas de transferencias, seguridad social, alimentación, guarderías y educación inicial. Otro lastre es que las mujeres en su etapa más productiva tienen menor acceso al trabajo remunerado por la maternidad (especialmente cuando los hijos se encuentran en la primera infancia), $79 \%$ de las mujeres de 25 a 44 años sin hijas e hijos contaban con un trabajo remunerado en el 2018, mientras que el porcentaje en las que eran madres fue de $61 \%$. También se encontró mayor incidencia de pobreza $(40 \%)$ conforme las jefaturas son de mujeres más jóvenes o bien de edad avanzada (CONEVAL, 2018b) (figura 3). 


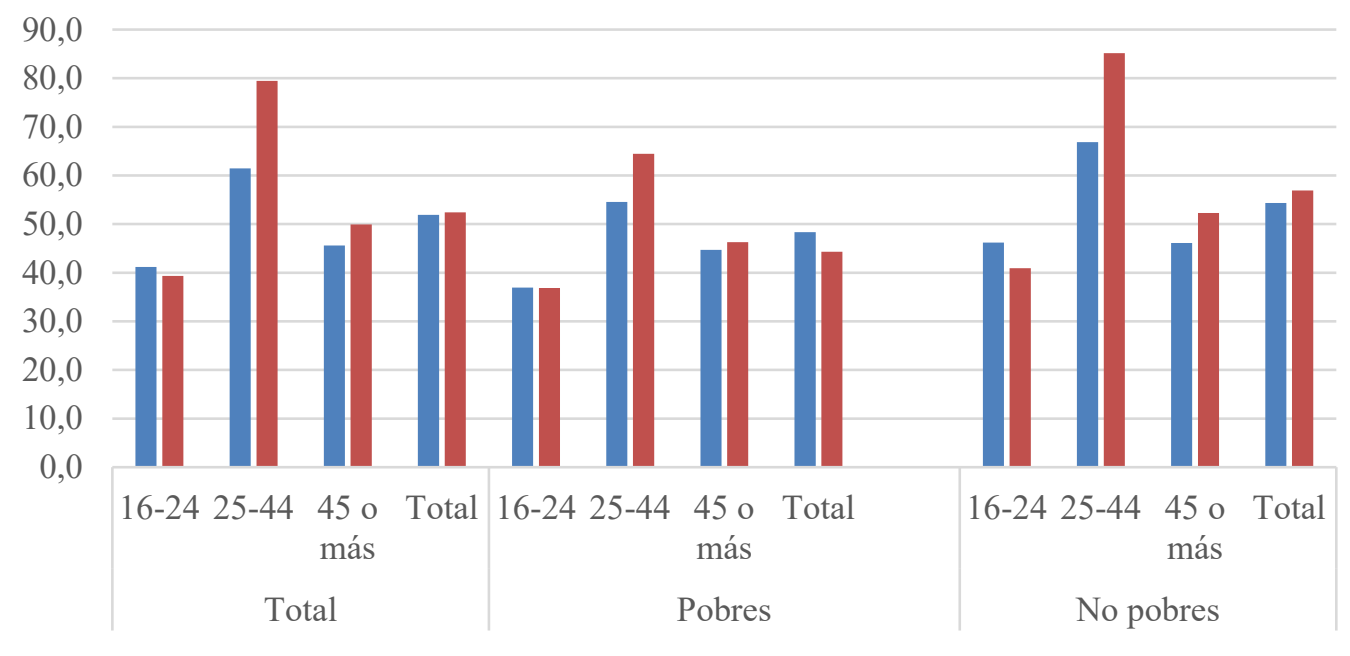

- Grupo de edad Mujeres con hijos/as a Grupo de edad Mujeres sin hijos/as

Figura 3. Porcentaje de participación económica femenina por maternidad, edad y

pobreza (2018)

Fuente: Base de Datos y Publicaciones Estadísticas de la CEPAL (2020).

Por lo anterior, es justamente la infancia en situación de pobreza la que más requiere una participación del Estado en la definición de políticas integradas de salud, nutrición, cuidado, protección, erradicación de la violencia y estimulación para el desarrollo. Todas ellas se podrían articular desde la Educación Inicial. Pero se requeriría la formación de cuadros técnicos y profesionales para cubrir dichos servicios de manera pertinente y acorde con las necesidades de las poblaciones más vulnerables. El balance de la situación de la primera infancia con datos duros al año 2018, refleja que la ley de protección de sus derechos es letra muerta.

\subsection{Cruce de la atención infantil con la pobreza de las mujeres}

Según estudios del Comité de Oxford de Ayuda contra el Hambre (OXFAM por sus siglas en inglés), los centros de Educación Inicial juegan un papel importante no solo por ofrecer un espacio exprofeso para el cuidado de niñas y niños, sino por su relación directa con la desigualdad económica de la mujer como consecuencia del sistema social patriarcal, que "favorece a unos cuantos a costa del trabajo gratuito o mal remunerado de millones de mujeres que cuidan a personas dependientes (principalmente niños) para que otros (generalmente hombres), se desempeñen laboralmente" (OXFAM-México, 2019, p. 1).

Del mismo modo, la brecha de género en trabajo no remunerado se ha incrementado en la última década (figura 4). Por cada 100 hombres ocupados sin pago, hubo 141 mujeres en 2018, lo cual representa una barrera importante para alcanzar la autonomía económica (CONEVAL, 2018b). 


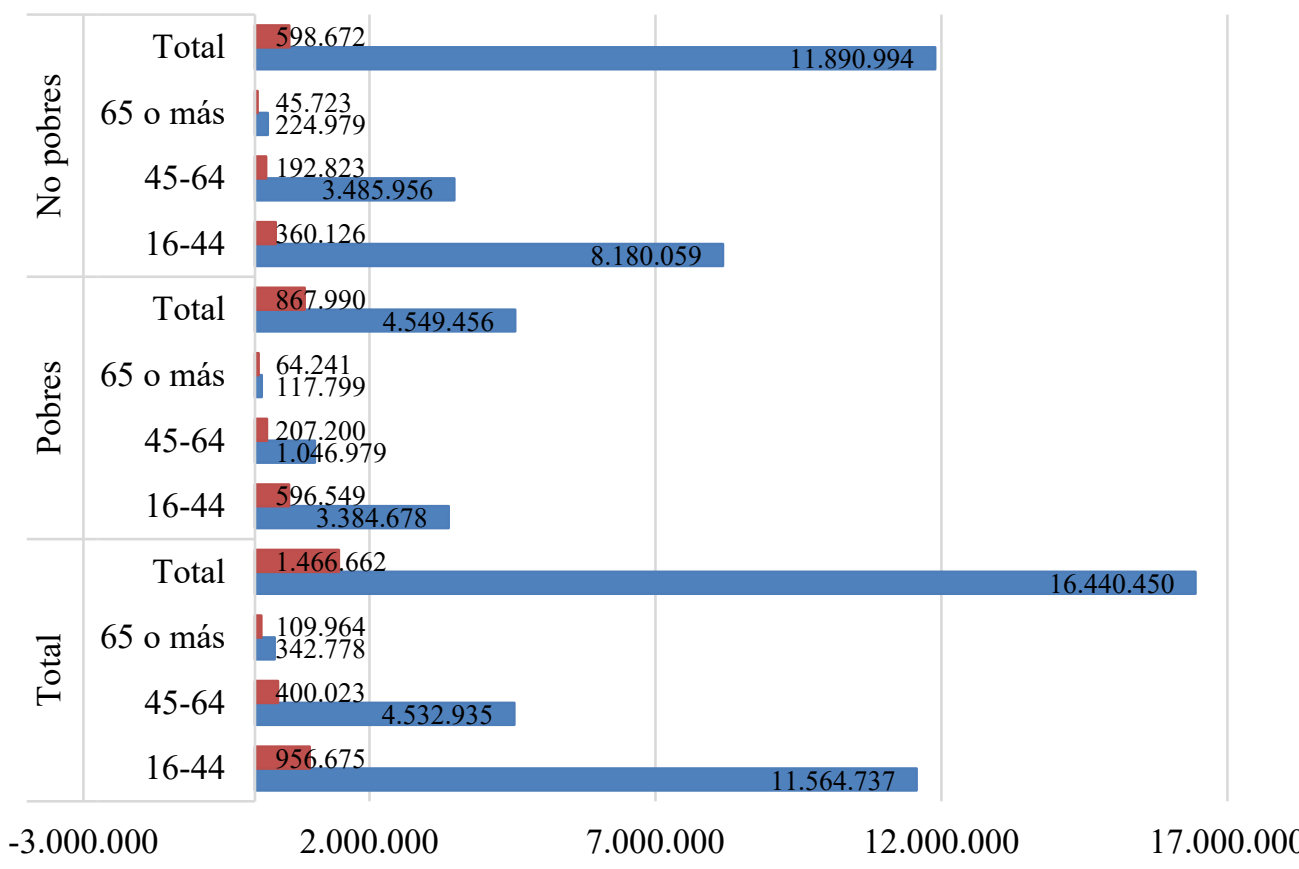

Grupo de edad Mujeres Sin pago $\quad$ Grupo de edad Mujeres Con pago

Figura 4. Mujeres ocupadas por grupos de edad y condición de pobreza (2018)

Fuente: Base de Datos y Publicaciones Estadísticas de la CEPAL (2020).

Una constante en la última década se refiere a la dedicación al trabajo doméstico y cuidado como actividad preponderantemente femenina: las mujeres destinan 2,5 veces más de su tiempo a los quehaceres del hogar que cualquier otro miembro de la familia, lo cual equivale en promedio a 22 horas a la semana a las tareas del hogar y 28 horas al cuidado de otras personas, mientras que los hombres dedicaron 8 y 15 horas respectivamente (CONEVAL, 2018b) (figuras 5 y 6).

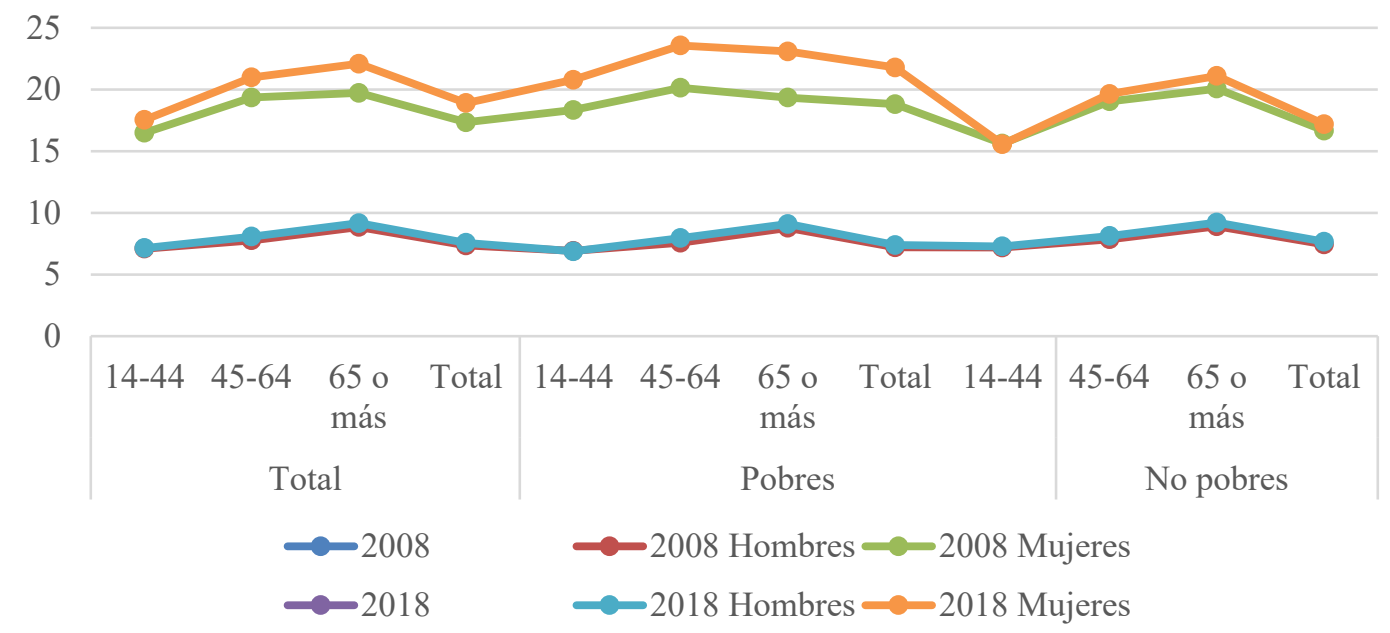

Figura 5. Tiempo promedio que hombres y mujeres ocupados/as destinan a actividades del hogar, por grupos de edad y condición de pobreza, 2008-2018 Fuente: Base de Datos y Publicaciones Estadísticas de la CEPAL (2020). 


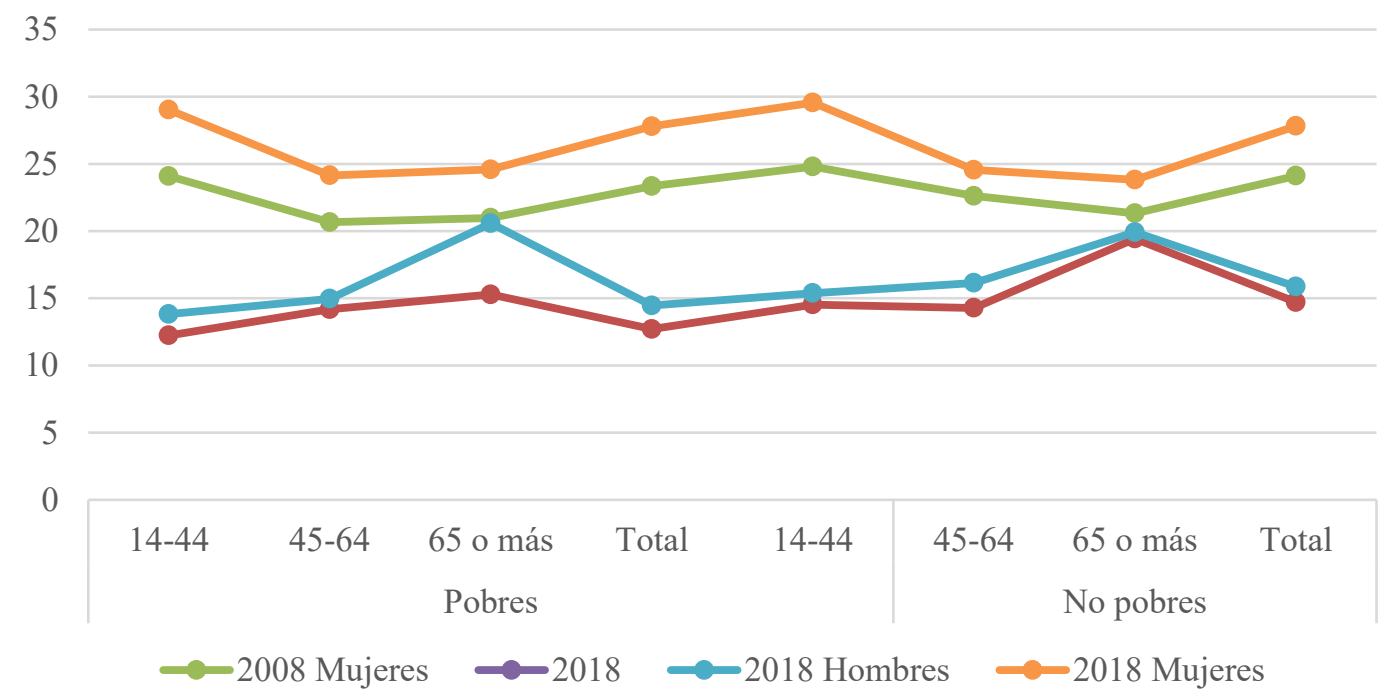

Figura 6. Tiempo promedio destinado al cuidado, por género, grupo de edad y condición de pobreza, 2008-2018

Fuente: Base de Datos y Publicaciones Estadísticas de la CEPAL (2020).

Un elemento que reproduce la inequidad del trabajo de las mujeres es la propensión a trabajar en actividades de cuidado y actividades domésticas para los hogares no pobres con bajas remuneraciones (El 98\% de las trabajadoras domésticas no tiene contrato, sólo $5 \%$ recibe vacaciones y el 15\% aguinaldo. OXFAM-México, 2019), lo cual no les permite alcanzar autonomía a partir del ingreso personal. De aquí la relevancia de considerar dentro de las encuestas de población la medición del tiempo dedicado al trabajo no remunerado dentro del hogar, ya que permite visibilizar el cuidado. Pero falta ponderar otros elementos que requiere el cuidado infantil que van más allá de la medición del tiempo invertido. Nos referimos a que no es suficiente estar físicamente presente al lado del niño, el adulto debe estar emocionalmente disponible para responder a las necesidades del infante y además promover su desarrollo pleno, lo cual lo hace un aspecto complejo para la medición.

Con las condiciones de pobreza, desigualdad y escasa cobertura infantil, resulta comprensible que la Ruta Integral de Acciones para la primera infancia (RIA) de 2018 (Secretaría de Gobernación, 2018) en el periodo presidencial de Enrique Peña Nieto, no apostó por la expansión de los servicios educativos, sino por la participación de los padres de familia de manera conjunta con el Programa del Instituto Mexicano del Seguro Social IMSS Bienestar en 79 hospitales rurales; también contó con la colaboración de UNICEF, la que desarrolló la fundamentación y materiales sobre cuidado parental y prácticas de crianza para niños de 0 a 5 años de las zonas con mayor marginación en México. Pero la RIA se ocupó básicamente del componente de salud, pero sin capacitar al personal en atención y desarrollo infantil, ni sobre prácticas positivas de crianza.

El convenio con UNICEF solamente incluyó la preparación sobre los mecanismos de protección especial y la restitución de derechos en 13 de las 32 Procuradurías de Protección estatales y programas piloto para prevenir la violencia en dos estados: Guerrero y Chihuahua (UNICEF, 2018). También con el respaldo de la UNICEF, se implementó el programa de Acogimiento Familiar como mecanismo de rescate de los 
niños que sufrían violencia intrafamiliar. Pero el programa solamente contó con la capacitación de 78 trabajadores sociales, psicólogos y abogados. lo que lo hizo insuficiente para la magnitud del problema; peor aún, solamente obtuvieron su certificación 17 familias de las 26 que participaron.

Otra línea en la protección de los derechos de los niños a cargo de la SIPINNA fue la Estrategia Nacional para la Prevención del Embarazo en Adolescentes (Enapea), institucionalizada en 2015, que se orientó a la erradicación del embarazo en niñas y adolescentes menores de 15 años y el matrimonio infantil, como metas a alcanzar en el año 2030. Pero no generó acuerdos de interacción con organizaciones de la sociedad y operó a través de un voluntariado conformado por 2,665 adolescentes comprometidas a difundir en sus comunidades una guía para la prevención del embarazo. La estrategia impacta en la primera infancia pues se ha comprobado que, a menor edad de las madres, menor escolaridad y mayor incumplimiento de los derechos de sus hijas e hijos. Aunado a esto, México posee la tasa más alta de incidencia en embarazo infantil y a menor edad entre los países de la OCDE (pues se han registrado embarazos en niñas desde los 10 años de edad); los estados que concentran el mayor porcentaje de embarazo en adolescentes son Coahuila (20.5\%), Chihuahua (20.4\%) y Durango (19.8\%) según datos del Instituto Nacional de Estadística, Geografía e Informática (2020), mientras que los que concentrar el mayor porcentaje de matrimonio infantil son Chiapas (44.82\%), Guerrero (42.41\%) y Oaxaca (39.17\%). Según el informe de UNICEF (2017), las entidades que no han prohibido el matrimonio en menores de edad sin excepción ni dispensa alguna son: Baja California, Chiapas, Chihuahua, Guanajuato, Guerrero, Nuevo León, Oaxaca, Querétaro, Tabasco y Sonora.

El proyecto se vinculó con el Centro Nacional de Equidad de Género y Salud Reproductiva (CNEGySR) y recibió respaldo de la Unión Nacional de Padres de Familia y ONU Mujeres en el marco de una estrategia global contra el matrimonio infantil. Estos resultados reflejan el incumplimiento de los acuerdos de la Décima Conferencia Regional sobre la Mujer de América Latina y el Caribe, conocida como el Consenso de Quito (CEPAL, 2007) respecto al compromiso de los Estados participantes de formular y aplicar políticas para favorecer la responsabilidad e importancia del cuidado como actividad compartida; así como asumir el cuidado y el bienestar de la población como objetivo de la economía y responsabilidad pública indelegable.

Finalmente, dentro de la dimensión normativa se destaca un caso excepcional de participación ciudadana exitosa. Se refiere a la iniciativa de ley promovida por los padres de familia que se constituyeron en la Asociación ABC (surgida a raíz del trágico deceso de 49 niños en una guardería subrogada del mismo nombre en el estado de Sonora en el año 2009), que impulsó la Ley para Regular la Prestación de Servicios para la Atención, Cuidado y Desarrollo Integral e Infantil, conocida como Ley 5 de junio, aprobada en la Cámara de Diputados federal, pero no homologada para su aplicación en todo el país. Por ello siguieron sin cumplirse los estándares de calidad del servicio, regulatorios ni sancionadores.

\subsection{La estrategia nacional de atención para la primera infancia en México}

Para Muller y Surel (1998), la tercera dimensión de análisis de las políticas públicas corresponde a las interacciones necesarias para la implementación, la atención y cuidado de los poco más de 12 millones de niños menores de cinco años. Para lograr lo anterior, se requiere de la participación del Estado, la sociedad, la familia y el mercado, claramente 
regulada y desde una concepción garantista del derecho de las niñas y niños desde el inicio de su vida. Aguilar (2015) señala que en torno a las políticas se construyen representaciones sociales, pero su formulación también se ve cruzada por barreras culturales como el clasismo o el género, que se traducen en determinadas ofertas diferenciadas de servicios. Estas ideas suelen determinar quién, cómo, cuándo y dónde deben ser atendidas las personas y va influyendo en la construcción de estereotipos y creencias sobre el cuidado.

El desafío en torno al cuidado de la niñez desde la perspectiva de la CEPAL, es su inclusión positiva en las políticas públicas para que el Estado cubra su función redistributiva, garantizando el derecho al bienestar de quienes no tienen la posibilidad de cubrirlo de otra manera. Resulta interesante que el cuidado infantil, la salud y la educación infantil empiezan a incluirse dentro de las políticas sociales por la sinergia que puede generar en la formación del capital humano en el corto y mediano plazo.

En la distribución de la responsabilidad social del cuidado el Estado, el mercado, las familias y diferentes actores de la sociedad (organizaciones formales e informales o comunitarias), tienen diferente nivel de participación de acuerdo con su identidad y afinidad ideológica. Pero con frecuencia ha recaído y recae más en las familias, lo que equivale a decir en la mayoría de los casos, en las mujeres de las familias. Su vinculación con las políticas de bienestar implica escalarlo a la altura de los derechos ciudadanos. Ese es uno de los caminos para garantizar su cobertura universal y hacerlo exigible.

La incorporación del cuidado a la agenda de la política pública no sólo es indispensable para romper con estereotipos de género, donde los imaginarios reducen a las mujeres a desempeñar roles asociados con la feminidad, la maternidad y el cuidado. También porque las oportunidades de acceso y calidad del cuidado están determinadas por la clase social. Tal vez la complejidad del tema del cuidado infantil:

Está anclad(o) en la multiplicidad de actores, instituciones y sectores que participan en el proceso de cuidado: se trata de diversos sectores de las políticas públicas (políticas de bienestar, educación, salud, etc.), de los servicios ofrecidos en el mercado, de todas las tareas domésticas visibles e invisibles, de la contribución de tareas realizadas a través de organizaciones sociales diversas, entre las cuales se cuentan -aunque no exclusivamentelas familias. Es claro desde el inicio que el tema sólo puede ser abordado desde una perspectiva intersectorial (Esquivel, Faur y Jelin, 2014, p. 12).

Respecto a la Inapea, la confluencia de diversos organismos nacionales e internacionales ha permitido valorar su impacto. En la evaluación de 2019, la organización Europa Social identificó que el programa tiene un impacto positivo al observarse un leve descenso de embarazo infantil al pasar de 74,3 nacimientos por cada mil adolescentes en el año 2015, a 69,5 en el año 2019 (Xantomila, 2020). Pero se requiere una actuación más decidida de los diferentes niveles de gobierno. De continuar esta tendencia, tomará 50 años más la erradicación del matrimonio y maternidad infantil en México.

Si bien el marco normativo avanza hacia la concepción garantista de los derechos de niñas y niños, en el discurso sigue presente la visión tutelar y conservadora respecto a la infancia al remitirlo como una cuestión a resolver en el ámbito privado de la familia. En el primer conversatorio Regional de América y el Caribe "En la ruta de la igualdad: 30 años de la Declaración de los Derechos del Niño”, el representante de México afirmó: 
La presentación se centró en la importancia de contar con un sistema de indicadores que considere a los abuelos que se hacen cargo de niños pequeños y diseñar, en consecuencia, sistemas de apoyo y acompañamiento dirigidos a esta población. Se relevó la importancia del tema de la primera infancia y destacó su carácter de categoría menor en el ámbito político. (CEPAL, 2018, p. 20)

En los últimos años, México ha tomado algunas medidas de seguridad social para paliar de alguna manera las consecuencias que han dejado las décadas del modelo neoliberal. El énfasis en las políticas de cuidado se ha centrado en la atención de los adultos mayores desde que Andrés Manuel López Obrador como Jefe de Gobierno instituyera la pensión universal para ellos. Si bien en su momento fue ampliamente cuestionado por el entonces presidente panista Vicente Fox Quezada, la medida fue replicada a nivel nacional, pero bajo un esquema de focalización para finalmente imponerse como programa universal. Dentro del bienestar, el cuidado debería ser considerado dentro de la seguridad social, por lo que debería ser analizada en toda su extensión, lo que implica que su atención intersectorial debe incluir además de los sectores de salud, educación, economía y trabajo.

La reforma constitucional del 15 de mayo de 2019 al inicio del sexenio presidencial de López Obrador, fijó la obligatoriedad de la educación inicial y estableció un plazo de 180 días para la elaboración de la Estrategia Nacional de Atención a la Primera Infancia (ENAPI). Hasta el año 2020 se incluye la concepción garantista del derecho de las niñas y niños al cuidado y educación hasta los 6 años de edad, acorde con el Marco de Cuidado Cariñoso y Sensible desarrollado por la Organización Mundial de la Salud (OMS), UNICEF y el Banco Mundial. Con ello, se cumple el Principio No.7 de los Objetivos para el Desarrollo Sostenible de terminar con la exclusión y no dejar a nadie atrás ni fuera del ejercicio de sus derechos. Con ello la ENAPI explicita el propósito de no deben perder de vista las limitaciones geográficas, históricas, económicas y culturales del país para la atención de la primera infancia.

Desde esta perspectiva, NN (niñas y niños) cuidados sensible y cariñosamente son aquellos que alcanzan niveles de cuidado que los mantienen protegidos, seguros, saludables y adecuadamente nutridos, con una atención sensible y receptiva que responde a sus intereses y necesidades, y los alienta a explorar su entorno e interactuar con sus cuidadores y otras personas relevantes. NN con estas características tienen menores probabilidades de incurrir en conductas de riesgo, y mayor potencialidad para aprender, relacionarse, ser más productivos, insertarse exitosamente en el mercado de trabajo y mejorar su ingreso (Secretaría de Educación Pública, 2020, p. 9).

Sin embargo, en los primeros meses de su gobierno, el presidente López Obrador decidió ordenar el cierre total de las estancias infantiles del programa subvencionado de guarderías para madres trabajadoras sin acceso a dicho servicio (garantizado en la ley desde 2006), por presuntos actos de corrupción. Tal decisión anticipó por la vía de los hechos un retorno a la concepción tutelar, familiar/privada del cuidado infantil y un desentendimiento por parte del Estado. Desafortunadamente el reconocimiento de derechos en la ley no es suficiente para garantizar el interés superior del niño. Es necesario crear sistemas de protección integral de la infancia, entendidos según Morlachetti (2013) como el conjunto de órganos, entidades, mecanismos e instancias a nivel nacional, regional y local orientados a respetar, promover, proteger, restituir y restablecer los derechos de niñas y niños, lo cual implica tomar en cuenta componentes de regulación e interacción entre instituciones públicas y privadas (del mercado y sociedad civil). 
La Estrategia divide la atención a la primera infancia en dos ciclos: el primer ciclo corresponde a la educación inicial (0 a 3 años) y el $2^{\circ}$ ciclo es cubierto por la educación preescolar (3 a 6 años). El documento reconoce que $51.9 \%$ de los niños de 0-6 años viven en condición de pobreza, "niños entre 0 y 1 año de edad son la población con mayor carencia de servicios de salud, mientras que aquéllos de 3 a 5 años tienen mayor carencia de servicios educativos" (Secretaría de Educación Pública, 2020 p. 34) También acepta que el enfoque de las políticas de atención no ha respetado los derechos de los niños, que ha sido precario, desarticulado en los distintos niveles de gobierno y que se carece de un sistema de información. Clasifica las brechas de acceso a los derechos básicos de los niños en cuatro tipos: salud y nutrición; educación y cuidados; protección infantil; y protección social y pobreza, con lo cual se reconocen los problemas, pero no apuesta por una participación decidida y garante del Estado. Por un lado, la Estrategia pretende garantizar el derecho a la educación en México desde el nacimiento desde una perspectiva de educación para la vida, y por otro, reduce su responsabilidad a concientizar sobre su importancia y no a garantizar su cobertura universal, pues la estrategia seleccionada consiste en campañas y acciones de difusión y orientación, con el apoyo de los sectores social y privado (no compromete ampliación de atención en el sector público).

Un elemento sustantivo (si se llega a concretar) será la creación de Sistema de Información sobre los Derechos de la Primera Infancia. Hasta el momento, la información disponible es escasa y dispersa. De superarse tal situación, se contaría con una base georreferenciada a nivel municipal para identificar las necesidades de la población infantil desde el nacimiento, y garantizar uno a uno sus derechos por ahora incumplidos. Baste mencionar dos ejemplos, en los casos de los derechos a la identidad y a la salud, en la actualidad sólo el 96\% de los recién nacidos obtiene su registro legal antes del año, y sólo el 34\% de los menores de tres años tiene cubierto el esquema de vacunación para evitar la muerte infantil por causas prevenibles. El gobierno mexicano reporta acciones sin una adecuada desagregación de la población de 0 a 5 años. Pareciera que los niños importan básicamente a partir de su incorporación a la escuela primaria.

La ENAPI tiene tres estrategias de atención, la universal (al igual que en programas anteriores), vuelve a privilegiar el componente de salud, pero se limita a lograr atención prenatal, registro al nacimiento y completar el esquema de vacunación.

La atención focalizada se centra en la identificación población infantil en segmentos vulnerables: pobreza, migración, desnutrición, embarazo adolescente o pertenencia indígena. La novedad consiste en considerar el estrés como factor que afecta el desarrollo potencial de los niños. Las estrategias incluyen transferencias monetarias, servicios educativos de guardería gratuitos o subvencionados, visitas domiciliarias a madres adolescentes.

La atención indicada, se restringe aún más, pues sólo se refiere a niños carentes de cuidado y protección, niños sin personas a cargo de ellos, en hogares violentos o con madres en depresión (Secretaría de Educación Pública, 2020), lo cual constituye un reto monumental en el país, según los datos presentados previamente. No se plantean acciones contundentes al respecto cuando se reconoce que la mitad de los niños sufre alguna forma de violencia y que solamente 13 de las 32 entidades del país cuentan con procuradurías especializadas para la atención de la infancia. 


\section{Conclusiones}

Los estudios sobre cuidado se han dado dentro de la crítica a la división sexual del trabajo, pensando en el derecho de la mujer a la autonomía económica, pero es necesario mover el enfoque a una concepción garantista de los derechos de las niñas y los niños; principalmente al cuidado como condición básica para asegurar su pleno desarrollo. En México no existe diseño de política pública con la pretensión de contrarrestar la división sexual del trabajo ni de garantizar plenamente el derecho de la infancia al cuidado por parte del Estado, tampoco para promover y supervisar la corresponsabilidad del mercado y la sociedad para alcanzarlo.

Las políticas de cuidado infantil reflejan las posiciones ideológicas, creencias e imaginarios de quien las establece. La Estrategia en México deja de manifiesto que la atención de la primera infancia para el gobierno en turno continúa en el terrero tutelar y familiarista, pues se compromete a orientar al sector privado (entiéndase a la familia) y al mercado sobre la importancia del cuidado, sin asumir estrategias directas que le permitan ampliar sus servicios.

En el diagnóstico se visibilizó a la primera infancia como grupo etario vulnerable, pero se invisibilizó de nuevo al carecer de acciones que le permitan interactuar con el mercado y la sociedad para su adecuada atención y cuidado, desoyendo la tendencia internacional a privilegiarla. No solamente por garantizar los derechos de los niños, sino por la conveniencia de "invertir" en este sector poblacional y por los beneficios que reporta a los individuos a lo largo de su vida y a la sociedad en su conjunto.

Establecer la obligatoriedad de la educación inicial y su estrategia de atención por sí misma no es suficiente para universalizar la cobertura de los derechos de los niños sin supeditarlos al derecho de las mujeres-madres-trabajadoras, ni como insumo para la escolarización posterior. Pero la prestación de servicios de educación inicial debe cumplir con estándares de prestación, supervisión y sanción en su marco regulatorio.

Al limitarse a campañas de orientación, la Estrategia Nacional para el Cuidado de la Primera Infancia del presidente López Obrador no posiciona la agenda de cuidado infantil en la sociedad mexicana, que vulnera constantemente a sus niñas y niños sin que el Estado garantice las condiciones de acceso a una vida digna, y sin asumir su corresponsabilidad en la protección, superando una visión individualista y particular de la familia (que se ve forzada a resolverlo por la vía del mercado, es decir, a pagar por el servicio).

Los informes sobre las condiciones de pobreza revelan impactos negativos particularmente en el cuidado de los niños, pues ambos padres suelen incorporarse al mercado laboral y sencillamente no hay quien se haga cargo de la atención oportuna de los hijos que en ocasiones quedan a cargo de otra niña. Esto debería obligar a los gobernantes a buscar estrategias creativas y solidarias para brindar una respuesta a tales necesidades.

Sacar el cuidado del ámbito familiar-privado continúa siendo un reto para garantizar el estado de bienestar de mujeres y niños. Si bien es indispensable la participación del mercado y la sociedad para alcanzar la cobertura universal de niñas y niños menores de cinco años, ello implica también que el Estado asuma su papel de garante de sus derechos y no abandone su función supervisora y sancionadora, velando siempre por el interés superior de niñas y niños. 


\section{Referencias}

Aguilar, L. F. (2015). Gobernanza y política pública para la igualdad. Consejo Nacional para Prevenir la Discriminación (CONAPRED).

Batthyany, K. (2015). Las políticas y el cuidado en América Latina. Una mirada a las experiencias regionales. CEPAL.

CEPAL. (2004). Entender la pobreza desde la perspectiva de género. CEPAL.

CEPAL. (2007). Consenso de Quito. CEPAL.

CEPAL. (2018). América Latina y el Caribe a 30 años de la aprobación de la convención sobre los derechos del niño. CEPAL.

CEPAL. (2020). Base de datos y publicaciones estadísticas de la CEPALSTAT. CEPAL.

Colín, A. R. (2014). La desigualdad de género comienza en la infancia. Red por los Derechos de la Infancia en México.

Consejo Nacional de Evaluación de la Política de Desarrollo Social. (2018a). Pobreza infantil y adolescente en México 2008-2016. Dónde vive y qué características tiene la población de o a 17 años en situación de pobreza. CONEVAL.

Consejo Nacional de Evaluación de la Política de Desarrollo Social. (2018b). Pobreza y género en México: hacia un sistema de indicadores. Información 2008-2018. CONEVAL.

Cruz, J. C. (2017). Con empleo informal, casi seis de cada 10 mexicanos. INEGI.

Daly, M. y Lewis, J. (2000). The concept of social care and the analysis of contemporary welfare states. British Journal of Sociology, 51(2), 281-298. https://doi.org/10.1111/j.1468-4446.2000.00281.x

Esquivel, V. (2011a). Sixteen years after Beijing: What are the new policy agendas for time-use data collection? Feminist Economics, 17(4), 215-238. https://doi.org/10.1080/13545701.2011.616947

Esquivel, V. (2011b). La economía del cuidado en América Latina: Poniendo a los cuidados en el centro de la agenda, Col. Atando cabos deshaciendo nudos. PNUD.

Esquivel, V., Faur, E. y Jelin, E. (2014). Las lógicas del cuidado infantil. Entre las familias, el estado y el mercado. UNICEF-IDES.

Faur, E. (2014). El cuidado infantil en el siglo XXI. Mujeres malabaristas en una sociedad desigual. Siglo XXI.

Florit, P. y Piedracueva, M. (2016). Mujeres y cuestión agraria: Capitalismo, explotación y patriarcado. Hemisferio Izquierdo, 7, 2-16.

Folbre, N. (1995). Holding hands at midnight: The paradox of caring labor. Feminist Economics, 1(1), 73-92. https://doi.org/10.1080/714042215

Gómez, M., García, L. y García, A. (2019) Crítica a la idea de infancia y proyecto educativo en las políticas públicas de Colombia. Revista Latinoamericana de Ciencias Sociales, Niñez y Juventud, 17(1), 153-168. https://doi.org/10.11600/1692715x.17109

Himmelweit, S. (1995). The discovery of unpaid work. Feminist Economics, 1(2),1-20. https://doi.org/10.1080/714042229

Instituto Nacional de Estadística, Geografía e Informática. (2020). Porcentaje de nacimientos registrados de madres adolescentes por entidad federativa de residencia habitual de la madre, 2010 a 2018. INEGI. 
Irwin, L., Arjumand, S. y Hertzman, C. (2007). Desarrollo de la primera infancia: Un potente ecualizador. University of British Columbia.

Jaramillo. L. (2007). Concepciones de infancia. Zona Próxima, 8, 108-123.

Larguía, I. y Dumoulin, J. (1976). Hacia una ciencia de la liberación de la mujer. Casa de las Américas, 17, 65-66. https://doi.org/10.2307/j.ctvt6rmbs.17

Legarreta, I. (2014). Cuidados y sostenibilidad de la vida: Una reflexión a partir de las políticas de tiempo. Papeles del CEIC, 1, 93-128. https://doi.org/10.1387/pceic.12427

Montaño, S. y Calderón, C. (2010). El cuidado en acción: Entre el derecho y el trabajo. CEPAL.

Morlachetti, A. (2013). Sistemas nacionales de protección integral de la infancia: Fundamentos jurídicos y estado de aplicación en América Latina y el Caribe. CEPAL.

Muller, P. y Surel, Y. (1998). L’annalyse des politiques publiques. Montchrétien.

OXFAM-México. (2019). Posicionamiento de OXFAM México sobre el trabajo del cuidado y desigualdad en México. OXFAM-México.

Pautassi, L. (2010). Cuidado y derechos: La nueva cuestión social. CEPAL.

Pautassi, L. (2012). Educación, cuidado y derechos. SER Social, 13(29), 10-24. https://doi.org/10.26512/ser_social.v13i29.12664

Presno, M., López. C. y Moyá, G. (2019). Políticas públicas de atención y educación de la primera infancia en América Latina. Una aproximación al estado del arte de las investigaciones y estudios 2013-2019. CEPAL.

Secretaría de Educación Pública. (2020). Estrategia nacional para la atención de la primera infancia en México. Secretaría de Educación Pública.

Secretaría de Gobernación. (2018). Programa nacional de prestación de servicios para la atención, cuidado y desarrollo integral infantil 2014-2018. Secretaría de Gobernación.

UNICEF. (2017) Informe anual. México 2017. UNICEF.

UNICEF. (2018) Informe anual. México 2018. UNICEF.

\section{Breve CV de la autora}

\section{Claudia Alaníz-Hernández}

Doctora en Educación por la Universidad Pedagógica Nacional y Profesora-Investigadora Titular de Tiempo Completo de la misma institución. Integrante del Sistema Nacional de Investigadores del CONACyT; de la Red Educativa Universitaria de Conocimiento y Acción Regional (REDUCAR) conformada por Universidades Pedagógicas de América Latina y el Caribe; y del Consejo Mexicano de Investigación Educativa (COMIE). Desarrolla como líneas de investigación las políticas de educación básica y la formación docente, sobre los cuales ha sido panelista en congresos nacionales e internacionales y publicado artículos en revistas científicas, capítulos de libros y libros. El último en coautoría sobre El estado de la formación de maestros de educación inicial en seis países de América Latina. ORCID ID: https://orcid.org/Oo00-0001-8269-7216. Email: calaniz@upn.mx 